\title{
Measuring the health related quality of life of people with ischaemic heart disease
}

\author{
M Dempster, M Donnelly
}

\begin{abstract}
Objectives-To inform researchers and clinicians about the most appropriate generic and disease specific measures of health related quality of life for use among people with ischaemic heart disease.

Methods-MEDLINE and BIDS were searched for research papers which contained a report of at least one of the three most common generic instruments or at least one of the five disease specific instruments used with ischaemic heart disease patients. Evidence for the validity, reliability, and sensitivity of these instruments was critically appraised.

Results-Of the three generic measures - the Nottingham health profile, sickness impact profile, and short form 36 (SF-36) - the SF-36 appears to offer the most reliable, valid, and sensitive assessment of quality of life. However, a few of the SF-36 subscales lack a sufficient degree of sensitivity to detect change in a patient's clinical condition. According to the best available evidence, the quality of life after myocardial infarction questionnaire should be preferred to the Seattle angina questionnaire, the quality of life index cardiac version, the angina pectoris quality of life questionnaire, and the summary index. Overall, research on disease specific measures is sparse compared to the number of studies which have investigated generic measures.

Conclusions-An assessment of the quality of life of people with ischaemic heart disease should comprise a disease specific measure in addition to a generic measure. The SF-36 and the quality of life after myocardial infarction questionnaire (version 2) are the most appropriate currently available generic and disease specific measures of health related quality of life, respectively. Further research into the measurement of health related quality of life of people with ischaemic heart disease is required in order to address the problems (such as lack of sensitivity to detect change) identified by the review.

(Heart 2000;83:641-644)
\end{abstract}

Keywords: quality of life; outcome measurement; ischaemic heart disease

An important aspect of service evaluation and development is the assessment of the nature and extent to which an intervention or treatment impacts on a patient's illness or condition and on their quality of life. Instruments designed to measure health related quality of life (HRQoL) can be divided into two categories-generic or disease specific. Generic measures provide a broad assessment of the health status of an individual and allow comparisons of HRQoL between groups of patients with different conditions. Often, these instruments are used to monitor progress and to assist in the distribution of resources. Disease specific instruments are designed to measure HRQoL by tapping those areas of life which may be affected by a specific condition or illness. Although these instruments are narrow in focus, they do have a couple of advantages over their generic counterparts. Firstly, disease specific instruments comprise domains or areas which are related closely to the areas of life explored by clinicians, and secondly, disease specific instruments tend to be more sensitive to detecting change in health status than generic instruments. ${ }^{1}$ As there are benefits with each type of instrument, it is recommended often that both should be used when evaluating HRQoL. However, a critical analysis of the properties of the growing range of generic and disease specific measures is necessary in order to guide and direct researchers and clinicians towards the most appropriate measures in terms of reliability, validity, and sensitivity to change.

\section{Generic instruments}

Several generic measures of HRQoL, or health status, have been used among people with ischaemic heart disease. The most commonly used generic instruments in heart disease are the Nottingham health profile (NHP) ${ }^{2}$ (used in approximately $40 \%$ of studies), the short form $36(\mathrm{SF}-36),{ }^{3}$ and the sickness impact profile $(\mathrm{SIP})^{4}$ (both used in approximately $24 \%$ of studies). Although there are other generic measures of HRQoL, this paper concentrates on reviewing the research evidence concerning the reliability, validity, and sensitivity of the three most commonly used instruments among this patient group.

The studies reviewed were obtained by a search of the MEDLINE and BIDS databases. Research which did not involve people with an ischaemic heart disease (for example, studies concerned solely with the impact on HRQoL of heart failure or heart transplantation) or which were devoted to the validation of a translated version of the instrument, were excluded from this review. The final list of studies reviewed included samples of patients who were described as having angina, myocardial infarction (MI), coronary artery disease, ischaemic heart disease, or patients who had 
undergone coronary artery bypass graft (CABG) or angioplasty. Thus, all of the studies reviewed in this paper have drawn their samples from the population of patients with ischaemic heart disease, as defined by the International Classification of Diseases, 10th revision (ICD-10).

Each article extracted from the literature search was examined for information about: reliability in the form of test-retest reliability coefficients and internal consistency coefficients; and sensitivity as indicated by reports of effect sizes (mean change between two points in time divided by baseline SD) or standardised response means (mean change between two points in time divided by SD of change scores). Evidence for validity was also sought in terms of whether an instrument appeared to be valid for use in discriminative or evaluative studies. ${ }^{5}$ For example, an instrument may be described as having good discriminative validity when patients with severe angina attain significantly higher scores than patients with mild angina, whereas an instrument with evaluative validity is likely to detect significant differences between pre- and post-CABG scores.

Only brief details of the content of each instrument are given, as several authors provide comprehensive reports of their content and development. ${ }^{67}$

NOTTINGHAM HEALTH PROFILE

Seventeen studies which used the NHP to measure HRQoL in ischaemic heart disease were reviewed. The NHP has been described as a useful survey tool in terms of assessing whether or not patients have a severe health problem but it does not provide a comprehensive measure of HRQoL. ${ }^{8}$ Nevertheless, it tends to be used for this purpose in practice. The NHP is divided into two parts. Part 1 requires a yes or no response to 38 statements, which are grouped into six scales: mobility, pain, energy, sleep, emotional reactions, and social isolation. Part 2 of the NHP asks about the effects of health on seven areas of daily life: work, looking after the home, social life, home life, sex life, interests and hobbies, and holidays. The NHP is short and can be administered very quickly because of the limited response choices.

Many studies use part 1 only of the NHP and so relatively little psychometric work has been done on part 2 . Results are inconsistent regarding the ability of the NHP scales to discriminate between the clinical classes of angina. This inconsistency may be because the clinical classification systems are not highly regarded as criterion measures. ${ }^{9}$ Results are also inconsistent regarding the ability of the NHP to discriminate between people with heart disease and healthy people.

All scales have moderate to high test-retest reliability but preintervention ceiling effects and poor responsiveness indices suggest that the NHP may be an inappropriate choice of instrument for evaluative studies. In summary, the NHP may be used to track large changes in health status such as pre- to postcardiac surgery but does not appear to be sensitive to smaller changes within groups. In particular, some scales, such as emotion, appear to have weak validity.

SHORT FORM 36

Ten studies which used the SF-36 to assess HRQoL among people with heart disease were reviewed. The SF-36 is perhaps the most well known and widely used generic health status measure. The 36 items on the questionnaire are grouped into eight scales: physical functioning, social functioning, role limitations caused by physical problems, role limitations caused by emotional problems, mental health, energy/vitality, bodily pain, and general health, and another single item which solicits a self assessment of health change over the past year.

Overall, the SF-36 appears to have good psychometric properties, though further research is required to investigate the sensitivity to change of the SF-36. There is no evidence to indicate that it can discriminate between classes of angina. However, some researchers and clinicians have warned against using the angina classification system as a criterion measure, as it may be insensitive to change, as mentioned previously. As an evaluative tool in the field of ischaemic heart disease, the mental health and general health scales do not appear to be responsive to change, and the role emotional and role physical scales are prone to ceiling effects. These scales may not measure specific changes that a patient with ischaemic heart disease experiences as his/her condition improves or deteriorates. So, results obtained from these four scales should be interpreted with caution.

SICKNESS IMPACT PROFILE

A review of nine studies which used the SIP to measure HRQoL among ischaemic heart disease patients was conducted. The SIP consists of 136 items grouped into 12 categories: sleep and rest, eating, work, home management, recreation and pastimes, ambulation, mobility, body care and movement, social interaction, alertness behaviour, emotional behaviour, and communication. Through cluster and factor analytical techniques three of these categories were further aggregated into a physical dimension (ambulation, mobility, and body care and movement) and four others into a psychosocial dimension (social interaction, alertness behaviour, emotional behaviour, and communication). The other five dimensions cannot be grouped in a coherent manner but an overall score for the SIP can be obtained. ${ }^{10}$

Internal consistency of the total SIP is high, but there is little information about the internal consistency of the 12 separate scales or the two separate dimensions. There is some information to support the discriminative validity of the physical and psychosocial dimensions and the total score of the SIP, but there is little to suggest that the 12 separate scales of the SIP would be of any value in a discriminative study. The same is true for test-retest reliability. The total SIP score appears to be responsive to changes in patients' health status after surgery but is not responsive to changes over time 
post-MI. The available evidence suggests that the SIP should not be separated into 12 scales but should be used to obtain a total score or scores for the physical and psychosocial dimensions when used in either discriminative or evaluative studies among patients with ischaemic heart disease.

COMPARISON OF GENERIC INSTRUMENTS

All three generic instruments cover the key areas of physical, social, and emotional functioning. However, the SF-36 and the NHP appear to have better content validity in the field of heart disease because they both cover the areas of energy/vitality and bodily pain. A measure of these domains would be appropriate and useful, if not essential, when exploring the HRQoL of people with heart disease.

A head to head comparison of the SIP and the NHP has been conducted among patients with angina $(73 \% \text { male, average age } 65 \text { years })^{11}$ and among post-MI patients (in two studies: $40 \%$ male, average age 72.7 years ${ }^{12} ; 81 \%$ male, average age 62 years $^{13}$ ). Little psychometric differences were found between the two instruments. It was suggested in all these studies that the SIP was slightly more sensitive, though it is much longer and so takes much more time to administer than the NHP.

The NHP has also been compared with the SF-36 for a sample of several types of patients (angina, post-MI and postcardiac surgery) undertaking a cardiac rehabilitation programme ( $57 \%$ male, mean age 57.1 years). The SF-36 had higher internal consistency coefficients and gave clearer evidence of discriminative validity than the NHP. ${ }^{14}$

\section{Disease specific instruments}

Research regarding disease specific HRQoL measures in heart disease was sparse before 1990. ${ }^{15}{ }^{16}$ Currently, there are approximately 10 instruments. The main ones are the quality of life after myocardial infarction (QLMI), ${ }^{17}$ the Seattle angina questionnaire (SAQ), ${ }^{18}$ the quality of life index (QLI)-cardiac version, ${ }^{19}$ the angina pectoris quality of life questionnaire (APQLQ) ${ }^{20}$ and the summary index. ${ }^{21}$

The MEDLINE and BIDS databases were searched using the names of each of the disease specific instruments listed above and the same exclusion criteria were used as outlined for the generic instruments.

QUALITY OF LIFE AFTER MYOCARDIAL INFARCTION The 26 items on the QLMI questionnaire are grouped into five domains: symptoms, restriction, confidence, self esteem, and emotion. ${ }^{22}$ Recently, assessments of the psychometric properties and a refinement of the content of the QLMI has been conducted. ${ }^{23}{ }^{24}$ The refined questionnaire (comprising the QLMI with two original questions removed and three new questions added) is known as the QLMI-2 and groups 27 items into three domains: emotional, physical, and social.

The QLMI-2 appears to have better psychometric properties than the original QLMI, as the domains of the QLMI-2 have higher internal consistency estimates and are based on fac- tor analysis. However, the evaluative properties of the QLMI-2 have yet to be investigated. Most of the QLMI domains have a moderate to strong evaluative dimension, submitting high estimates of test-retest reliability and moderate to high responsiveness indices. As there is little difference in the content of the QLMI and the QLMI-2, it is likely that the QLMI-2 would be at least equally as useful as the QLMI in evaluative studies.

SEATTLE ANGINA QUESTIONNAIRE

The SAQ consists of 19 items, which are grouped into five separate domains: physical limitation, anginal stability, anginal frequency, treatment satisfaction, and disease perception. It is described by the authors as a disease specific functional status measure but seven out of the 19 questions tap social and emotional issues, which makes the SAQ definable as a measure of disease specific HRQoL.

All SAQ domains appear to be psychometrically sound. However, the treatment satisfaction and anginal stability domains may not be suitable for evaluative purposes, as the responsiveness estimate of the former and the test-retest reliability estimates of the latter are very low. Perhaps we should not expect treatment satisfaction to improve along with an improvement in the other SAQ domains. It is possible that a patient's health can improve and their HRQoL can improve, but their satisfaction with treatment may not increase any further. It could also be that the anginal stability scale is very sensitive to change in clinical condition and so perhaps this domain will still identify minor changes in a patient who is defined as stable by other measures.

QUALITY OF LIFE INDEX-CARDIAC VERSION The QLI was first designed for use with dialysis patients ${ }^{19}$ but has been adapted for use with other groups, including cardiac patients. The most recent form of the QLI to be used with cardiac patients is the QLI-cardiac version III, which contains 72 items. These items are divided into two equal parts: part 1 measures the satisfaction of patients with various life domains; and part 2 measures the importance of these domains to the patient. Scores on part 1 are weighted by the responses on part 2 . Four domains are covered by the 72 items: health and functioning, socioeconomic, psychosocial/ spiritual, and family.

Although the version of the QLI discussed here has been designed specifically for cardiac patients, the QLI was not developed originally for use with this population. In addition, it has not been used often with cardiac patients. Therefore, it is unsurprising that further work is required to investigate the discriminative and evaluative value of the QLI-cardiac version.

\section{ANGINA PECTORIS QUALITY OF LIFE}

QUESTIONNAIRE

The APQLQ has 22 items, which are divided into four scales: physical activities, somatic symptoms, emotional distress, and life satisfaction. The APQLQ has good psychometric properties for discriminative purposes. However, fur- 
ther work, such as an examination of responsiveness and test-retest reliability, is required.

\section{SUMMARY INDEX}

The APQLQ has been combined with the anginal impact questionnaire ${ }^{21}$ and the psychological general well being scale ${ }^{25}$ to produce a summary index for health related quality of life assessment in angina. The anginal impact questionnaire and the psychological general well being scale both consist of 22 items. The summary index, which combines all three questionnaires, consists of 51 items and can be divided into six categories: impact of angina on daily life, physical exertion, vitality, alertness, self control, and emotional function.

The summary index has received relatively little research attention. However, the reliability and responsiveness properties of the instrument appear to be good. Further evidence to support the validity of the summary index is required. The length of the index and its lack of "user friendliness", overall, may make it difficult to apply in clinical practice.

COMPARISON OF DISEASE SPECIFIC INSTRUMENTS A head to head comparison of the SAQ and the QLI-cardiac version has been conducted among patients with angina (all male, average age 65 years). ${ }^{26}$ The SAQ was able to discriminate between patients in Canadian Cardiovascular Society (CCS) classes I-III, whereas the QLI could not. The SAQ was also found to be slightly more sensitive to clinical changes than the QLI.

No other head to head comparisons of these instruments have been undertaken. This is an area of need in the research if we are to be able to pick the most appropriate disease specific HRQoL instrument in a given situation.

\section{Conclusions}

Any review of HRQoL instruments faces the same problem - finding an agreed criterion or "gold standard" against which each instrument can be judged. In the absence of a criterion, HRQoL instruments are often validated against clinical measures of health. This is not wholly satisfactory but it does allow us to judge the validity of the HRQoL instruments in relative terms, as is the case in this review, and clinical measures are meaningful to the practitioner. The psychometric evidence suggests that the SF-36 is the best available generic measure of HRQoL among people with ischaemic heart disease, though some of the SF-36 scales may not be useful in evaluative studies. The SF-36 is the shortest of the three generic measures reviewed, which makes it more acceptable to patients, clinicians, and service providers.

The best disease specific measures appear to

website

extra

Additional article outlining the measures of health related quality of life appears on the Heart website

www.heartjnl.com evidence, a more detailed psychometric analysis of the QLMI-2 has been conducted, when compared to the SAQ, and so it is the recommended choice of disease specific patients who have ischaemic heart disease.

This review makes it apparent that some aspects of generic instruments may not be sen- sitive or perhaps relevant to the specific problems faced by ischaemic heart disease patients. There are also aspects of the disease which impact on a person's life in ways which are not measured by the generic instruments. In order to measure change in HRQoL more accurately and to explore those domains which are affected particularly by ischaemic heart disease, a disease specific measure of $\mathrm{HRQoL}$ provides a useful, if not a necessary, addition to the generic instrument.

This work was supported by a grant from the Northern Ireland Chest Heart and Stroke Association.

1 Tullis DE, Guyatt GH. Quality of life in cystic fibrosis. Pharmacoeconomics 1995;8:23-33.

2 Hunt SM, McKenna SP, McEwen J, et al. A quantitative approach to perceived health status: a validation study. $\mathcal{F}$ Epidemiol Community Health 1980;34:281-6.

3 Ware JE, Snow KK, Kosinski MK, et al. SF-36 health survey manual and interpretation guide. Boston, MA: The Health Institute, New England Medical Center; 1993.

4 Bergner M, Bobbitt RA, Pollard WE, et al. The sickness impact profile: validation of a health status measure. Med Care 1976;14:57-67.

5 Fletcher AE, Bulpitt CJ. Measurement of quality of life in clinical trials of therapy. Cardiology 1988;75(suppl 1):41-52.

6 Bowling A. Measuring health. A review of quality of life measurement scales. Milton Keynes, UK: Open University Press, 1991.

7 Walker SR, Rosser RM. Quality of life: assessment and application. Lancaster: MTP Press, 1988.

8 McDowell I, Newell C. Measuring health: a guide to rating scales and questionnaires. New York: Oxford University

9 Bowling A. Measuring disease. Buckingham, UK: Open University Press, 1995.

10 Bergner $M$. Development, testing and use of the sickness impact profile. In: Walker SR, Rosser RM, eds. Quality of life: assessment and application. Lancaster, UK: MTP Press, 1988:79-94.

11 Visser MC, Fletcher AE, Parr G, et al. A comparison of three quality of life instruments in subjects with angina pectoris: the sickness impact profile, the Nottingham health profile, and the quality of well being scale. 7 Clin Epidemiol 1994;47:157-63.

12 Visser MC, Koudstaal PJ, Erdman RA, et al. Measuring quality of life in patients with myocardial infarction or stroke: a feasibility study of four questionnaires in The Netherlands. $\mathcal{F}$ Epidemiol Community Health 1995;49:513-17.

13 Taylor R, Kirby B, Burdon D, et al. The assessment of recovery in patients after myocardial infarction using three generic quality-of-life measures. $f$ Cardiopulm Rehabil 1998;18:139-44.

14 Dempster M, Bradley J, Wallace E, et al. Measuring quality of life in cardiac rehabilitation: comparing the short form 36 and the Nottingham health profile. Coronary Health Care 1997;1:211-17.

15 Mayou R. Quality of life in cardiovascular disease. Psychother Psychosom 1990;54:99-109.

16 Avis NE, Smith KW, Hambleton RK, et al. Development of the multidimensional index of life quality: a quality of life measure for cardiovascular disease. Med Care 1996;34: 1102-20.

17 Oldridge N, Guyatt GH, Jones N, et al. Effects of quality of life with comprehensive rehabilitation after acute myocardial infarction. Am f Cardiol 1991;67:1084-9.

18 Spertus JA, Winder JA, Dewhurst TA, et al. Development and evaluation of the Seattle angina questionnaire-a new functional status measure for coronary artery disease. $f \mathrm{Am}$ Coll Cardiol 1995;25:333-41.

19 Ferrans CE, Powers MJ. Quality of life index: development and psychometric properties. Adv Nurs Sci 1985;8:15-24.

20 Wiklund I, Comerford MB, Dimenäs E. The relationship between exercise tolerance and quality of life in angina pectoris. Clin Cardiol 1991;14:204-8.

21 Wilson A, Wiklund I, Lahti T, et al. A summary index for the assessment of quality of life in angina pectoris. $\mathcal{F}$ Clin Epidemiol 1991;44:981-8.

22 Hillers TK, Guyatt GH, Oldridge N, et al. Quality of life after myocardial infarction. F Clin Epidemiol 1994;47:128796

23 Lim LL-Y, Valenti LA, Knapp JC, et al. A self administered quality of life questionnaire after acute myocardial infarction. f Clin Epidemiol 1993;46:1249-56.

24 Valenti L, Lim L, Heller RF, et al. An improved questionnaire for assessing quality of life after acute myocardial infarction. Quality of Life Research 1996;5:151-61. HRQoL measurement instrument for use with
25 Dupuy HJ. The psychological general well-being index. In: Assessment of quality of life in clinical trials of cardiovascular therapies. Wenger NK, M

26 Dougherty CM, Dewhurst T, Nichol WP, et al. Comparison of three quality of life instruments in stable angina pectoris: Seattle angina questionnaire, short form health survey (SF-36), and quality of life index-cardiac version III. $\mathcal{f}$ Clin Epidemiol 1998;51:569-75. Assessment of quality of life in clinical trials of cardiovascular 